\title{
On the Performance Comparison of VSF-OFCDMA and OFDMA
}

\author{
Chia-Chin Chong, Fujio Watanabe and Hiroshi Inamura \\ DoCoMo Communications Laboratories USA, Inc. \\ 3240 Hillview Avenue, Palo Alto, CA 94304 \\ Email: \{cchong, watanabe, inamura\}@docomolabs-usa.com \\ Dandan Wang, Hlaing Minn and Naofal Al-Dhahir \\ Dept. of Electrical Engineering, \\ University of Texas at Dallas, Richardson, TX 75083 \\ Email: $\{\mathrm{dxw} 053000$, hxm025000, aldhahir $@$ utdallas.edu
}

\begin{abstract}
${ }^{1}$ Abstract-In this paper, we compare the downlink capacity and throughput between the variable spreading factor-orthogonal frequency and code division multiple access (VSF-OFCDMA) with one-cell frequency reuse, and the orthogonal frequency division multiple access (OFDMA) with conventional three-cell frequency reuse. Since the multiuser diversity gain in OFDMA systems can be achieved by approximately designing its access schemes, while it is non-trivial for the VSF-OFCDMA systems, the effect of both access schemes on the link capacity are investigated. Our simulation results reveal that although the multiuser diversity gain achieved by OFDMA systems can compensate the loss of low frequency reuse at low to mid range of signalto-interference-plus-noise ratio (SINR) values, the achievable throughput of VSF-OFCDMA systems is much higher than the OFDMA systems. Furthermore, the impact of user's location on the inter-cell-interference is also reported.
\end{abstract}

\section{INTRODUCTION}

The orthogonal frequency division multiplexing (OFDM) technology is well known for its capability to cope with frequency-selective fading channel due to multipath and its low complexity equalizer. Therefore, it becomes a popular candidate for various high data-rate wireless systems such as IEEE 802.11n WLAN, IEEE 802.16 WiMAX, 3GPP LTE, 3GPP2 UMB, etc. However, OFDM suffers from high peakto-average-power-ratio (PAPR). Furthermore, in multi-cell scenarios, the OFDM based access scheme, namely, orthogonal frequency division multiple access (OFDMA) can suffer severe co-channel interference from the adjacent cells which makes one-cell frequency reuse non-trivial for OFDMA based cellular networks. On the other hand, code division multiple access (CDMA) is a technology being used in the $3 \mathrm{G}$ cellular networks such as 3GPP WCDMA and 3GPP2 CMDA2000. The most significant advantage of CDMA is that it can achieve the one-cell frequency reuse among adjacent cells by deploying a properly designed set of codes or pseudo-noise sequence. Later, several technologies combining the benefits of OFDM with CDMA are reported in the literature, mainly in the form of multi-carrier CDMA (MC-CDMA) [1]-[3]. In particular, the variable spreading factor orthogonal frequency and code

\footnotetext{
${ }^{1}$ This work was done when D. Wang was an intern in DoCoMo USA Labs.
}

division multiplexing (VSF-OFCDM) which belong to the family of MC-CDMA was first proposed by NTT DoCoMo [3]. It was shown in [4] and [5] as a very promising broadband packet wireless access scheme in order to achieve very high data-rate using an approximate bandwidth of 50 to $100 \mathrm{MHz}$. The main advantage of VSF-OFCDM is due to its flexibility in which the spreading factor can be varied according to the cell structure and radio link conditions, which makes it more suitable to be used from multi-cell to isolated-cell environments while maintaining high link capacity.

The superiority of VSF-OFCDM over OFDM in a multicell environment, such as a cellular system has been shown in [6] and [7]. However, [6] and [7] mainly consider the peak throughput of a cell without considering the effects of the access scheme. Researchers in [8] and [9] shown that multiple diversity gain can be achieved in a system if the access scheme is properly designed. The basic idea of multiuser diversity is to exploit the randomness of fading channels among different users and to allocate the channel to the user with the largest channel power gain. The larger the dynamic range of channel fluctuations and the number of users, the larger the achievable multiuser diversity gain.

In OFDMA systems, users are scheduled to transmit on orthogonal frequency bands which enables the exploitation of multiuser diversity in the frequency domain since users are scheduled to transmit on their frequency fading peaks as proposed in [10] and [11]. On the other hand, in basic setting of VSF-OFCDMA systems, users are scheduled to transmit on orthogonal spreading codes over all frequency bands, which makes it difficult to achieve the multiuser diversity gain. Due to these reasons, it is important to investigate the performance of VSF-OFCDMA and OFDMA under the effects of the multiuser diversity gains.

The remainder of this paper is organized as follows: In Section II, the main features of VSF-OFCDMA and OFDMA are reviewed. In Section III, the system simulation configuration is described. Simulation results are presented in Section IV. Finally, Section V concludes the paper. 


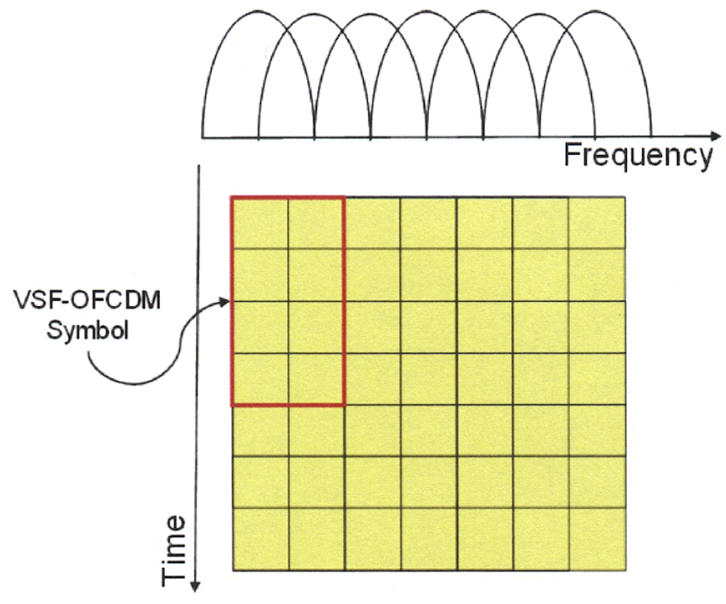

Fig. 1. Illustration of the VSF-OFCDM symbol.

\section{COMPARISON OF VSF-OFCDMA AND OFDMA}

\section{A. VSF-OFCDMA}

In VSF-OFCDMA systems, users access the system with orthogonal spreading factor using VSF-OFCDM. VSF-OFCDM spreads the data in both the time and frequency domains using orthogonal short channelization codes as shown in Fig. 1. The total spreading factor, $S F$ is $S F=S F_{t} \times S F_{f}$, where $S F_{t}$ and $S F_{f}$ are the spreading factors in the time and frequency domain, respectively. The time domain spreading is superior over the frequency domain spreading in order to maintain the orthogonality among the code-multiplexed channels with less inter-code interference. In particular, this property is advantageous for the application of adaptive modulation coding (AMC) scheme since the channel does not vary too much in the time domain. On the other hand, in order to reduce the effects of co-channel interference from adjacent cells in the case of one-cell frequency reuse (see Fig. 2(a)), a larger spreading factor can be used in addition to a cell-specific long scrambling code which is used to whiten the interference.

\section{B. OFDMA}

In OFDMA systems, the sub-carriers are divided into several groups and each group, namely, sub-channel is allocated to one user. Different techniques have been proposed in the literature to group these sub-carriers such as adjacent grouping (i.e., adjacent sub-carriers are grouped into one sub-channel), distributed grouping (i.e., distributed sub-carriers are grouped into one sub-channel), etc. In this paper, we focus on the adjacent grouping technique so that it is more pronounced to see the effect of multiuser diversity gain and the benefit of utilizing AMC.

There are rich literature about the scheduling schemes in OFDMA systems in order to achieve the multiuser diversity gain such as [10]-[12] and the references therein. Here, in order to perform fair comparison with VSF-OFCDMA, we consider the case in which the number of users is equal to the number of sub-channels, and each sub-channel is allocated to one user. In the rest of the paper, we will use the following three scheduling schemes to illustrate the impact of scheduling schemes on the system performance:

1) Ideal Scheduling: Each user is scheduled to transmit on its best sub-channel on each frame. Note that, this scheme is too optimistic and impractical since more than one user could choose the same best sub-channel.

2) Fixed Scheduling: Each user is scheduled to transmit on a specific sub-channel, which is fixed over all frames. Note that, no multiuser diversity gain is achieved in this scheduling scheme.

3) Random Scheduling: Each randomly selected user is scheduled to transmit on its best available sub-channel. For example, the first randomly selected user is scheduled to transmit on its best sub-channel which will not be reused by other users. Then, the second randomly selected user (excluding the user which has been scheduled a subchannel) is scheduled to transmit on its best available subchannels. The same process continues until all the users are being scheduled with their respective sub-channels.

\section{Simulation Configuration}

\section{A. System Level Simulation Setup}

In the system level simulation, a 19-hexagonal cell layout with one sector per cell is assumed for both wireless access schemes. In practice, usually a fixed and limited amount of bandwidth are available. Therefore, in order to have a fair comparison between VSF-OFCDMA and OFDMA, the same amount of bandwidth will be used by both schemes. Here, one-cell frequency reuse will be deployed in VSF-OFCDMA and while three-cell frequency cell reuse will be deployed in OFDMA as illustrated in Fig. 2. Base stations (BSs) are located at the center of each cell, and the mobile stations (MSs) are distributed uniformly over the whole cell. Here, we assume that each MS is only connected to one target BS, from which the MS's received the strongest signal power among all the BSs. The 3GPP Spatial Channel Model (SCM) is used in the system level simulations. This model has a distance-dependent path loss and lognormal shadowing as reported in [13]. In this paper, only the urban-micro scenario is being considered. The system level simulation parameters are summarized in Table I. The average received signal-to-interference-plus-noise-ratio (SINR) at each MS is defined as the ratio of the received signal power from the target BS to the sum of the received signal power from other cells and the background noise.

\section{B. Link Level Simulation Setup}

In the link level simulation, the 3GPP SCM multipath channel model is used and only one cell is considered [13]. The throughput achieved by a user in this cell is measured as a function of the received SINR. The link level simulation parameters are summarized in Table II. Note that since the three-cell frequency reuse is deployed in OFDMA scheme, the bandwidth of each cell (i.e., the number of sub-carriers) is $1 / 3$ of that in VSF-OFCDMA scheme that deployed onecell frequency reuse. In VSF-OFCDMA, the total spreading 


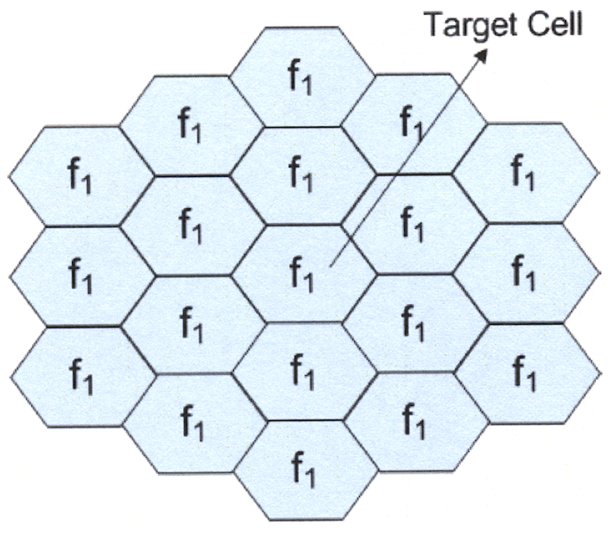

(a) VSF-OFCDMA: one-cell frequency reuse

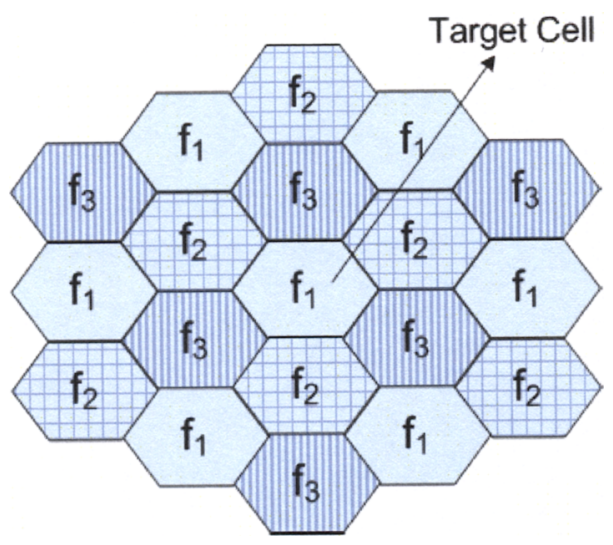

(b) OFDMA: three-cell frequency reuse

Fig. 2. Cell layout for (a) VSF-OFCDMA with one-cell frequency reuse, and (b) OFDMA with three-cell frequency reuse.

TABLE I

System LeVel Simulation Parameters

\begin{tabular}{|l|l|}
\hline Cellular layout & 19 cells, 1 sector/cell \\
\hline $\begin{array}{l}\text { Distance dependent path } \\
\text { loss and shadowing }\end{array}$ & $\begin{array}{l}\text { 3GPP SCM [13], Urban- } \\
\text { micro scenario }\end{array}$ \\
\hline Radius, $r$ & $500 \mathrm{~m}, 1000 \mathrm{~m}, 1500 \mathrm{~m}$ \\
\hline Carrier frequency & $2 \mathrm{GHz}$ \\
\hline Background thermal noise & $-98 \mathrm{dBm}$ \\
\hline BS transmit power & $43 \mathrm{dBm}$ \\
\hline BS antenna height & $32 \mathrm{~m}$ \\
\hline MS antenna height & $1.5 \mathrm{~m}$ \\
\hline MS velocity & $10 \mathrm{~m} / \mathrm{s}$ \\
\hline MS distribution in a cell & Uniformly distributed \\
\hline
\end{tabular}

factor is $S F=16$ in which only the time domain spreading is applied i.e., $S F_{t}=16$ and $S F_{f}=1$. The applied modulation and channel coding scheme are summarized in Table III. Here, we assumed that perfect channel knowledge are available at the receiver.
TABLE II

LiNK LEVEl Simulation PARAMETERS

\begin{tabular}{|c|c|c|c|}
\hline \multicolumn{2}{|l|}{ System } & VSF-OFCDMA $^{a}$ & OFDMA $^{b}$ \\
\hline \multicolumn{2}{|c|}{ Bandwidth } & $101.5 \mathrm{MHz}$ & $34.0 \mathrm{MHz}$ \\
\hline \multicolumn{2}{|c|}{ No. of sub-carriers } & 768 & 256 \\
\hline \multicolumn{2}{|c|}{ Spreading factor ${ }^{c}$} & 16 & - \\
\hline \multicolumn{2}{|c|}{ No. of subchannels } & 768 & $256 / 16=16$ \\
\hline \multirow{2}{*}{$\begin{array}{l}\text { Spreading } \\
\text { Code }\end{array}$} & Scrambling & Pseudo random $^{d}$ & \multirow[t]{2}{*}{-} \\
\hline & Short & $\begin{array}{l}\text { Walsh- } \\
\text { Hadamard }\end{array}$ & \\
\hline \multicolumn{2}{|c|}{$\begin{array}{l}\text { Symbol duration } \\
\text { (Data + guard interval) }\end{array}$} & \multicolumn{2}{|c|}{$\begin{array}{c}9.259 \mu \mathrm{s} \\
(7.585+1.674) \mu \mathrm{s}\end{array}$} \\
\hline \multicolumn{2}{|c|}{ Frame length } & \multicolumn{2}{|c|}{$\begin{array}{c}0.481 \mathrm{~ms} \\
(48 \text { data }+4 \text { pilot symbols })\end{array}$} \\
\hline \multicolumn{2}{|c|}{$\begin{array}{l}\text { Channel coding/ } \\
\text { decoding }\end{array}$} & \multicolumn{2}{|c|}{$\begin{array}{c}\text { Convolutional coding } \\
\text { Viterbi decoding } \\
\end{array}$} \\
\hline \multicolumn{2}{|c|}{ Number of active users } & \multicolumn{2}{|c|}{$\begin{array}{l}\{1,2, \cdots, 16\} \text { uniformly } \\
\text { distribution in each cell }\end{array}$} \\
\hline \multicolumn{2}{|c|}{ Channel model } & \multicolumn{2}{|c|}{ 3GPP SCM, urban-micro scenario } \\
\hline
\end{tabular}

${ }^{a}$ One-cell frequency reuse.

${ }^{b}$ Three-cell frequency reuse.

${ }^{c} S F=S F_{t} \times S F_{f}=S F_{t} \times 1$.

${ }^{d} 768$ chips/sub-carriers

C. Impact of Users' Location

In one-cell frequency reuse VSF-OFCDMA systems, the inter-cell interference (ICI) can be reduced by deploying the cell-specific long scrambling code. However, the residual ICI will still have impact on the system performance. In this paper, we investigate the effect of the ICI when a MS is at a different location within the cell. In particular, we consider a MS moves with $10 \mathrm{~m} / \mathrm{s}$ along the horizonal line from the center of a target cell to the cell edge. Here, we define the target cell as the center cell of the 19-hexagonal cell layout (see Fig. 2). The cell radius, $r$ is $1000 \mathrm{~m}$ and QPSK modulated symbols with channel coding rate $R=\frac{1}{2}$ are transmitted. The distances to the target cell are $0.1 r, 0.3 r, 0.5 r, 0.7 r$, and $0.9 r$, respectively.

\section{Simulation Results}

\section{A. Throughput Comparison of VSF-OFCDMA and OFDMA}

Fig. 3 compares the cumulative distribution function (CDF) of the average received SINR for VSF-OFCDMA and OFDMA systems. It can be seen that VSF-OFCDMA has larger probability of small SINR value than OFDMA especially when $r$ is small. This is due to the fact that threecell frequency reuse is deployed in the latter and thus, it experiences much smaller ICI than the former which deploy one-cell frequency reuse. Interestingly, when the $r$ increases, the differences between VSF-OFCDMA and OFDMA become insignificant. For example, at $r=1500 \mathrm{~m}$, both schemes have almost identical performances. This is because when the $r$ is 
TABLE III

Modulation and Channel Coding Schemes

\begin{tabular}{|l|l|l|l|}
\hline \multicolumn{2}{|c|}{ VSF-OFCDMA } & \multicolumn{2}{c|}{ OFDMA } \\
\hline $\begin{array}{l}\text { Modulation \& } \\
\text { coding }\end{array}$ & $\begin{array}{l}\text { Data } \\
\text { rate } \\
(\mathrm{Mbps})\end{array}$ & $\begin{array}{l}\text { Modulation \& } \\
\text { coding }\end{array}$ & $\begin{array}{l}\text { Data } \\
\text { rate } \\
(\mathrm{Mbps})\end{array}$ \\
\hline QPSK, R=1/2 & 4.8 & QPSK, $\mathrm{R}=1 / 2$ & 1.6 \\
\hline QPSK, R=3/4 & 7.2 & QPSK, $\mathrm{R}=3 / 4$ & 2.4 \\
\hline 16QAM, R=1/2 & 9.6 & 16QAM, R=1/2 & 3.2 \\
\hline 16QAM, R=3/4 & 14.4 & 16QAM, $\mathrm{R}=3 / 4$ & 4.8 \\
\hline 64QAM, R=1/2 & 14.4 & 64QAM, R=1/2 & 4.8 \\
\hline 64QAM, R=2/3 & 19.2 & 64QAM, $\mathrm{R}=2 / 3$ & 6.4 \\
\hline 64QAM, R=3/4 & 21.6 & 64QAM, $\mathrm{R}=3 / 4$ & 7.2 \\
\hline
\end{tabular}

large, only users at the cell edge will experience significant ICI. On the other hand, when $r$ is small, even users located close to the center of the cell might also experience severe ICI.

Fig. 4 shows the throughput comparison for VSF-OFCDMA and OFDMA with different scheduling schemes as described in Section II. The throughput is achieved by using the modulation and coding schemes given in Table III. As we can see, from low to mid range of SINR values (i.e., $<25$ dB), VSF-OFCDMA performs better than OFDMA with fixed scheduling, but perform slightly worst when ideal and random scheduling schemes are used in OFDMA scheme. This is mainly due to multiuser diversity gain brought by the latter two scheduling schemes which compensate the effect of low frequency reuse. However, when the received SINR is high (i.e., $>25 \mathrm{~dB}$ ), VSF-OFCDMA achieves much higher throughput than OFDMA irrespective of the scheduling schemes used as illustrated in Fig. 4.

The CDF of the achievable throughput as shown in Fig. 5 can be obtained from Figs. 3 and 4. Different from the previous works reported in [6] and [7] in which only the peak throughput of a cell are shown, here, the "throughput" is defined as the achievable throughput for a user using only one sub-channel (i.e., $1 / 16$ of the total available resources). From Fig. 5, we can see that when $r=500 \mathrm{~m}$, the probability that the throughput is less than 1 Mbps for VSF-OFCDMA and OFDMA (with ideal, fixed, and random scheduling schemes) are approximately $66 \%, 25 \%, 36 \%$, and $30 \%$, respectively. When $r$ increases, the difference between VSF-OFCDMA and OFDMA at low achievable throughput become smaller (see Fig. 5(c)). However, irrespective to the value of $r$, in general, VSF-OFCDMA achieves much higher throughput than OFDMA. For example, the highest throughput achieved by VSF-OFCDMA is about 20 Mbps while it is only about 7 Mbps for OFDMA i.e., approximately three times better.

\section{B. Performance Comparison at Different Users' locations}

Fig. 6 shows the packet error rate (PER) and Fig. 7 shows the throughput comparison for VSF-OFCDMA and OFDMA when a user is at different locations. It can be seen that when the user moves away from the center of the target cell, the

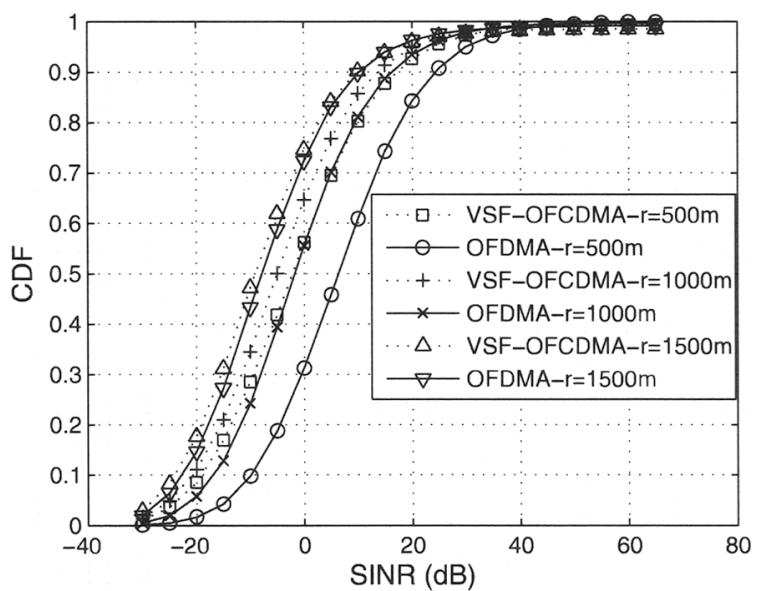

Fig. 3. CDF of the average received SINR for VSF-OFCDMA and OFDMA.

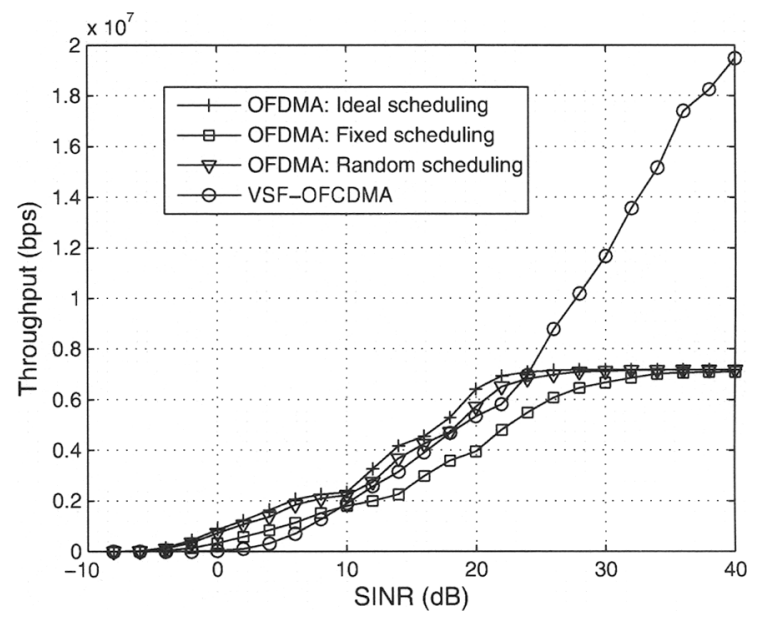

Fig. 4. Throughput comparison of VSF-OFCDMA and OFDMA with different scheduling schemes.

performance degrades with increased in PER and decreased in throughput for both schemes. When the user is located close to the center of the target cell, VSF-OFCDMA achieves much higher throughput than OFDMA due to its three times larger bandwidth utilization. However, when the user is close to the cell edge, OFDMA performs better than VSF-OFCDMA due to three-frequency reuse. Note that for the analysis in this paper, we fixed the spreading factor to $S F=16$. We believe that by using longer spreading factor, the performance of VSFOFCDMA for users at the cell edge could be further improved by reducing the effect of ICI. However, this investigation will leave open as our future work.

\section{CONCLUSION}

In this paper, we have compared the performance of two widely used broadband multiple access schemes for high data-rate applications, namely, VSF-OFCDMA and OFDMA. Different from works already reported in the literature, here, 


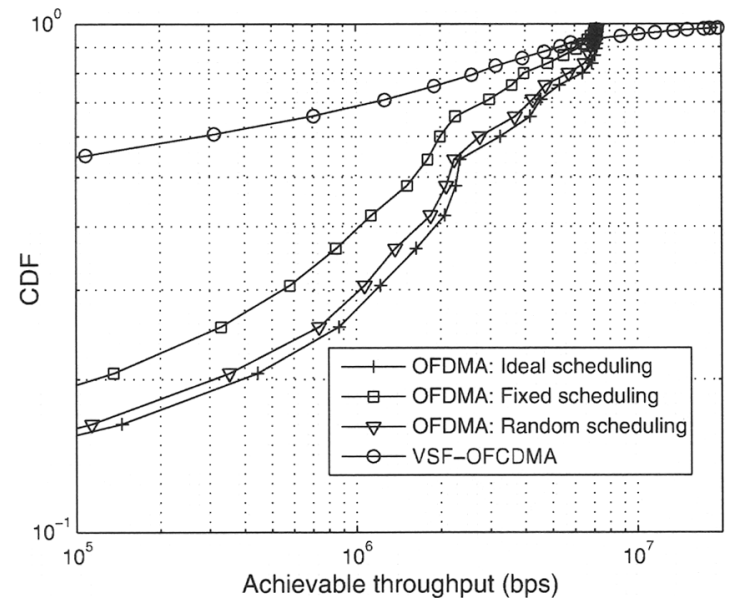

(a) $r=500 \mathrm{~m}$

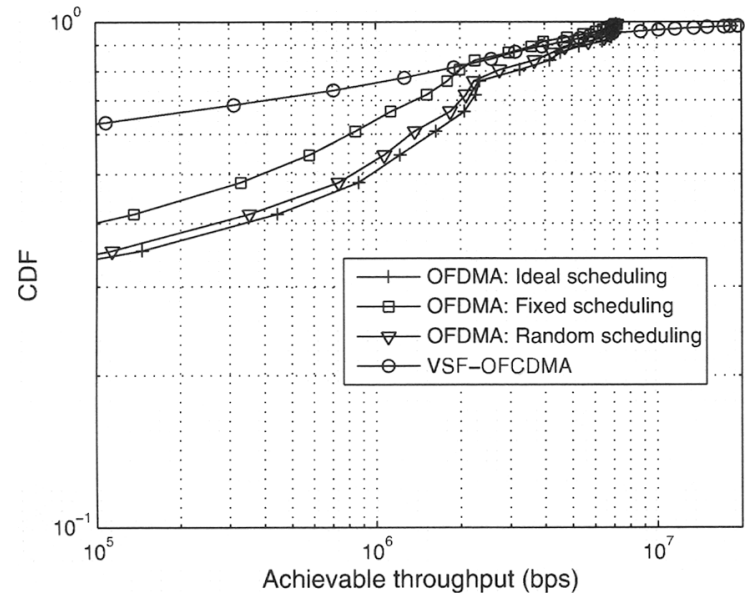

(b) $r=1000 \mathrm{~m}$

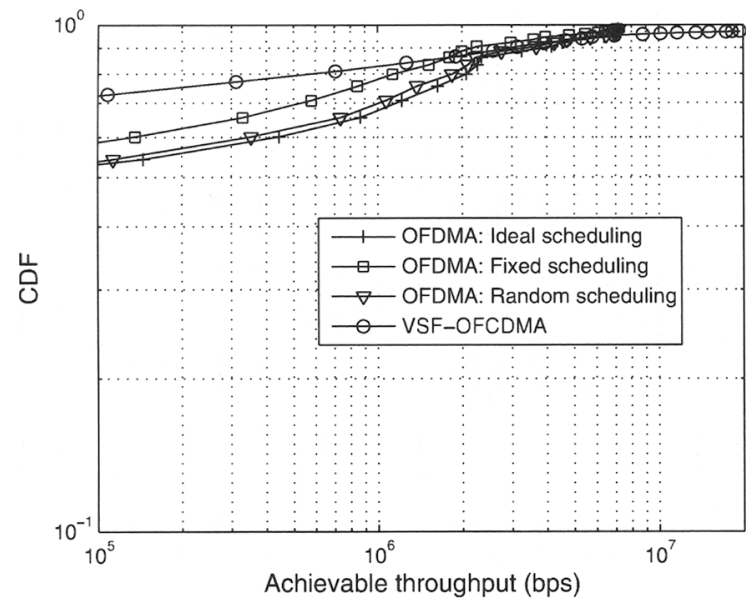

(c) $r=1500 \mathrm{~m}$

Fig. 5. CDF of the achievable throughput for (a) $r=500 \mathrm{~m}$, (b) $r=1000$ $\mathrm{m}$, and (c) $r=1500 \mathrm{~m}$.

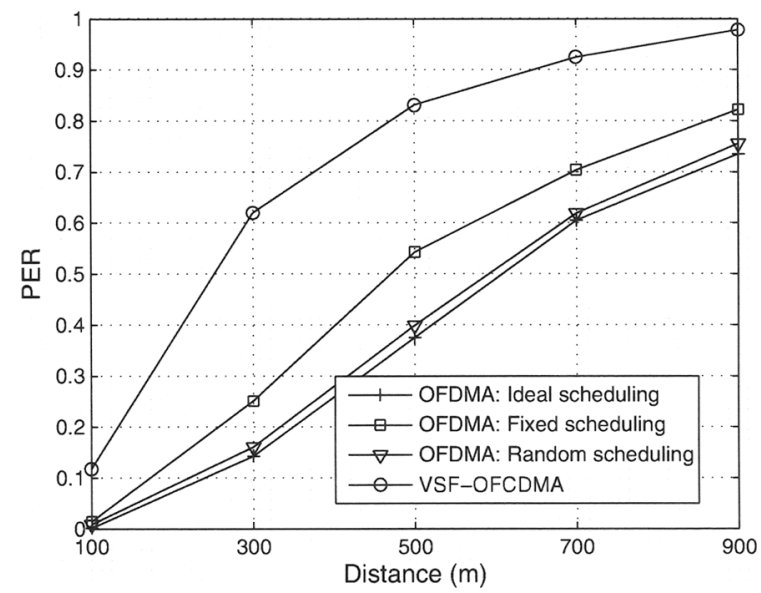

Fig. 6. PER comparison of VSF-OFCDMA and OFDMA at different user's location.

we mainly focus on the impact of these two access schemes on system performance such as throughput, received SINR, impact of user's location on PER and throughput. Our study reveal that at low to mid range of SINR values, the multiuser diversity gain achieved by OFDMA can compensate the effect of low frequency reuse such that OFDMA systems can achieve comparable throughput as VSF-OFCDMA systems. However, at high SINR values, the throughput performance of VSFOFCDMA systems is superior over the OFDMA systems. In particular, we consider a scenario of uniformly distributed user in which the highest throughput achievable by VSFOFCDMA is about three times more than the OFDMA. This implies that, for broadband wireless systems, it is possible to deploy hybrid multiple access schemes such as the combination of VSF-OFCDMA and OFDMA in order to provide high throughput from low to high SINR region. Future work includes studying the impact of different spreading factors and designing improved scheduling schemes that can achieve multiuser diversity gain for the VSF-OFCDMA systems.

\section{REFERENCES}

[1] N. Yee, J.-P. Linnartz, and G. Fettweis, "Multi-carrier CDMA in indoor wireless radio networks," in IEEE Pers. Indoor Mobile Radio Commun., Sept. 1993, pp. 109-113.

[2] K. Fazel and L. Papke, "On the performance of convolutional-coded CDMA/OFDM for mobile communication systems," in IEEE Pers. Indoor Mobile Radio Commun., Sept. 1993, pp. 468-472.

[3] H. Atarashi and M. Sawahashi, "Variable spreading factor orthogonal frequency and code division multiplexing (VSF-OFCDM)," in 2001 Third International Workshop on Multi-Carrier Spread Spectrum (MCSS 2001) Related Topics, 2001.

14] S. Abeta, H. Atarashi, M. Sawahashi, and F. Adachi, "Performance of coherent multi-carrier/DS-CDMA and MC-CDMA for broadband packet wireless access," in IEICE Trans. Commun., vol. E84-B, no. 3, Mar. 2001, pp. 406-414.

[5] H. Atarashi, S. Abeta, and M. Sawahashi, "Broadband packet wireless access appropriate for high-speed and high-capacity throughput," in IEEE Veh. Techn. Conf., May 2001, pp. 566-570.

[6] Y. Kishiyama, N. Maeda, K. Higuchi, H. Atarashi, and M. Sawahashi, "Experiments on throughput performance above 100-Mbps in forward 


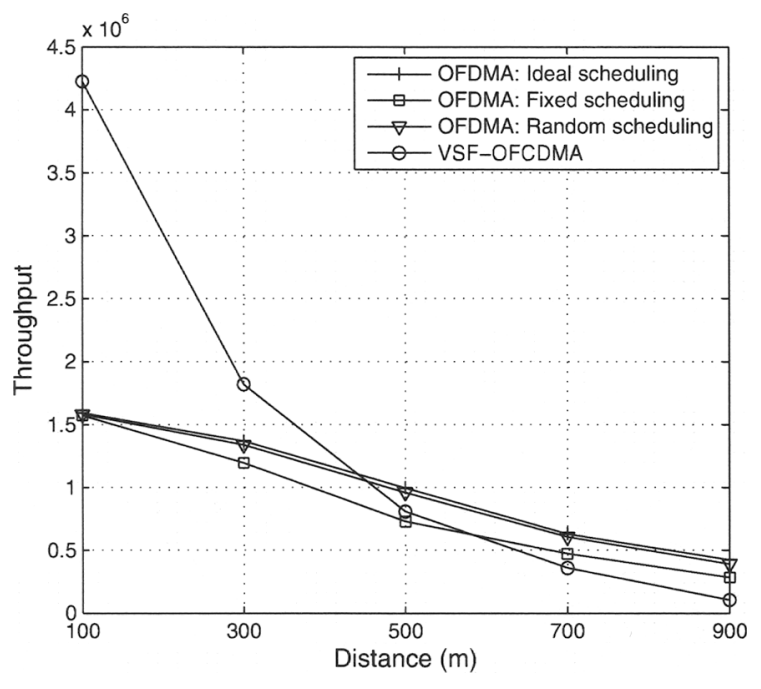

Fig. 7. Throughput comparison of VSF-OFCDMA and OFDMA at different user's location. link for VSF-OFCDM broadband wireless access," in IEEE Veh. Techn. Conf., Oct. 2003, pp. 1863-1868.

[7] N. Maeda, H. Atarashi, S. Abeta, and M. Sawahashi, "Throughput comparison between VSF-OFCDM and OFDM considering effect of sectorization in forward link broadband packet wireless access," in IEEE Veh. Techn. Conf., Sept. 2002, pp. 47-51.

[8] R. Knopp and P. A. Humblet, "Information capacity and power control in single-cell multiuser communications," in IEEE Int. Conf. Commun. Seattle, WA, Jun. 1995, pp. 331-335.

[9] D. N. C. Tse, "Optimal power allocation over parallel gaussian channels," in IEEE Int. Symp. Information Theory, Ulm. Germany. Jun. 1997, pp. 22-27.

[10] M. Sternad, T. Ottosson, A. Ahln, and A. Svensson, "Attaining both coverage and high spectral efficiency with adaptive OFDM," in IEEE Veh. Techn. Conf., Oct. 2003, pp. 2486-2490.

[11] W. Wang. T. Ottosson, M. Sternad, A. Ahln, and A. Svensson, "Impact of multiuser diversity and channel variability on adaptive OFDM," in IEEE Veh. Techn. Conf., Oct. 2003. pp. 547-551.

[12] P. Svedman, S. K. Wilson, and B. Ottersten, "A QoS-aware proportional fair scheduler for opportunistic OFDM," in IEEE Veh. Techn. Conf., Sept. 2004, pp. 558-562.

[13] 3rd Generation Partnership Project (3GPP), "Technical specification group radio access network; Spatial channel model for Multiple Input Multiple Output (MIMO) simulations (Release 7)," Tech. Rep. 